\title{
Reinforced Limit of a MEMS Model with Heterogeneous Dielectric Properties
}

\author{
Philippe Laurençot ${ }^{1} \cdot$ Katerina Nik $^{2} \cdot$ Christoph Walker ${ }^{2}$
}

Published online: 14 May 2020

(c) The Author(s) 2020

\begin{abstract}
A MEMS model with an insulating layer is considered and its reinforced limit is derived by means of a Gamma convergence approach when the thickness of the layer tends to zero. The limiting model inherits the dielectric properties of the insulating layer.
\end{abstract}

Keywords MEMS - Transmission problem - Gamma convergence · Mixed boundary conditions · Non-Lipschitz domains

Mathematics Subject Classification 35Q74 · 74G65 · 35J20 · 35J25

\section{Introduction}

Idealized microelectromechanical systems (MEMS) consist of two dielectric plates: a rigid ground plate above which an elastic plate is suspended. The latter is electrostatically actuated by a Coulomb force which is induced across the device by holding the two plates at different voltages. In this set-up there is thus a competition between attractive electrostatic forces and restoring mechanical forces due to the elasticity of

Partially supported by the CNRS Projet International de Coopération Scientifique PICS07710.

$\bowtie$ Christoph Walker

walker@ifam.uni-hannover.de

Philippe Laurençot

laurenco@math.univ-toulouse.fr

Katerina Nik

nik@ifam.uni-hannover.de

1 Institut de Mathématiques de Toulouse, UMR 5219, Université de Toulouse, CNRS, 31062

Toulouse Cedex 9, France

2 Institut für Angewandte Mathematik, Leibniz Universität Hannover, Welfengarten 1, 30167 Hannover, Germany 


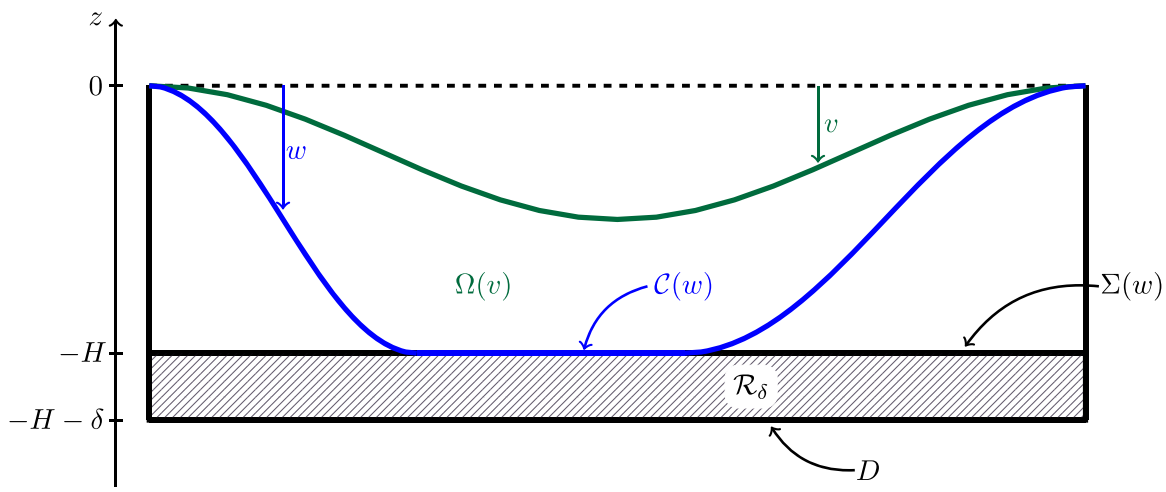

Fig. 1 Geometry of $\Omega_{\delta}(u)$ when $n=1$ for a state $u=v$ with empty coincidence set (green) and a state $u=w$ with non-empty coincidence set (blue)

the plate. When the two plates are not prevented from touching each other, a contact of the plates commonly leads to an instability of the device-also known in the literature as "pull-in instability"-which is revealed as a singularity in the corresponding mathematical equations, e.g., see $[9,16]$ and the references therein. In contrast, when the ground plate is coated with an insulating layer preventing a direct contact of the plates, see Fig. 1, a touchdown of the elastic plate on this layer does not result in an instability as the device may continue to operate without interruption (though it still leads to a peculiar situation from a mathematical point of view). Different mathematical models describing this setting including an insulating layer were introduced $[2,4,10,12,13,17]$. The basic assumption in all these models is that the state of the device is fully described by the vertical deflection of the elastic plate and the electrostatic potential in the device. According to $[4,10,12,13]$ the dynamics of the former is governed by an evolution equation while that of the latter is governed by an elliptic equation in a time-varying domain enclosed by the two plates. Due to the heterogeneity of the dielectric properties of the device, this elliptic equation is actually a transmission problem (see (1.1) below) on the non-smooth time-dependent domain with a transmission condition at the interface separating the insulating layer and the free space. The analysis of such a model turns out to be quite involved [10, Section 5]. Therefore, several simpler and more tractable models were derived on the assumption of a vanishing aspect ratio of the device $[2,4,10,12,13]$. Thanks to this approximation the electrostatic potential can be computed explicitly in terms of the deflection of the elastic plate, and the model thus reduces to a single equation for the deflection.

The aim of the present work is to derive an intermediate model by letting only the thickness of the insulating layer go to zero (instead of the aspect ratio of the device). Our starting point is the model analyzed in [10] in which we introduce an appropriate scaling of the dielectric permittivity in dependence on the layer's thickness (see (2.1a) below) and use a Gamma convergence approach to study the limiting behavior. The specific choice of the scaling is required in order to keep relevant information of the dielectric heterogeneity of the device and can be interpreted as a reinforced limit from a mathematical point of view [1]. 
To be more precise, we recall the model stated in [10, Section 5]. Let $D \subset \mathbb{R}^{n}$ with $n \geq 1$ be a bounded $C^{2}$-domain representing the (identical) horizontal crosssection of the two plates (actually, only the cases $n \in\{1,2\}$ are physically relevant for applications to MEMS, the ground plate being $D \times(-H-d,-H)$ and thus a two or three dimensional object). The dielectric layer of thickness $\delta>0$ on top of the ground plate located at $z=-H-\delta$ with $H>0$ is then given by

$$
\mathcal{R}_{\delta}:=D \times(-H-\delta,-H)
$$

The deflection of the elastic plate from its rest position at $z=0$ is described by a function $u: \bar{D} \rightarrow[-H, \infty)$ with $u=0$ on $\partial D$, so that

$$
\Omega(u):=\{(x, z) \in D \times \mathbb{R}:-H<z<u(x)\}
$$

is the free space between the elastic plate and the top of the dielectric layer. We let

$$
\Sigma(u):=\{(x,-H): x \in D, u(x)>-H\}
$$

denote the interface separating free space and dielectric layer and put

$$
\Omega_{\delta}(u):=\{(x, z) \in D \times \mathbb{R}:-H-\delta<z<u(x)\}=\mathcal{R}_{\delta} \cup \Omega(u) \cup \Sigma(u) .
$$

If the elastic plate and the insulating layer remain separate, that is, if $u>-H$ in $D$, then $\Sigma(u)$ coincides with

$$
\Sigma:=D \times\{-H\}
$$

In contrast, a touchdown of the elastic plate on the insulating layer corresponds to a non-empty coincidence set

$$
\mathcal{C}(u):=\{x \in D: u(x)=-H\}
$$

and a different geometry as the free space $\Omega(u)$ then has several connected components. It is worth pointing out that in this case - independent of the smoothness of the function $u$-these components may not be Lipschitz domains, a feature which requires some special care in the mathematical analysis.

The different situations with empty and non-empty coincidence sets are depicted in Fig. 1.

In the model considered in [10, Section 5], the deflection $u$ of the elastic plate is governed by an evolution equation involving contributions from mechanical and electrostatic forces, the latter depending on the electrostatic potential denoted by $\psi$ in the following. However, for the derivation of the limiting problem for the electrostatic potential, the evolution of $u$ does not play any role. We thus consider throughout this paper a fixed geometry $\Omega(u)$; that is, we consider the function $u: \bar{D} \rightarrow[-H, \infty)$ with $u=0$ on $\partial D$ describing the deflection of the elastic plate as given and fixed. We refer to [10, Section 5] for the full model. 
Given such a function $u$, the electrostatic potential $\psi=\psi_{u, \delta}$ satisfies the transmission problem

$$
\begin{aligned}
\operatorname{div}\left(\sigma_{\delta} \nabla \psi\right) & =0 \quad \text { in } \Omega_{\delta}(u), \\
\llbracket \psi \rrbracket=\llbracket \sigma_{\delta} \partial_{z} \psi \rrbracket & =0 \quad \text { on } \Sigma(u), \\
\psi & =h_{u, \delta} \quad \text { on } \partial \Omega_{\delta}(u),
\end{aligned}
$$

and the corresponding electrostatic energy of the device with geometry $\Omega_{\delta}(u)$ is

$$
E_{e, \delta}(u):=-\frac{1}{2} \int_{\Omega_{\delta}(u)} \sigma_{\delta}\left|\nabla \psi_{u, \delta}\right|^{2} \mathrm{~d}(x, z)
$$

Here, $\sigma_{\delta}$ is the permittivity of the device, which is different in the insulating layer and free space, and $h_{u, \delta}$ is a given suitable function describing the boundary values of the electrostatic potential. By $\llbracket \cdot \rrbracket$ we denote the jump of a function across the interface $\Sigma(u)$. The Lax-Milgram theorem provides the existence of a unique electrostatic potential $\psi_{u, \delta}=\chi_{u, \delta}+h_{u, \delta}$ solving (1.1) in a variational sense, and the function $\chi_{u, \delta} \in H_{0}^{1}\left(\Omega_{\delta}(u)\right)$ is the minimizer of the Dirichlet integral

$$
G_{\delta}[\vartheta]:=\frac{1}{2} \int_{\Omega_{\delta}(u)} \sigma_{\delta}\left|\nabla\left(\vartheta+h_{u, \delta}\right)\right|^{2} \mathrm{~d}(x, z)
$$

among functions $\vartheta \in H_{0}^{1}\left(\Omega_{\delta}(u)\right)$, see Proposition 3.2 below.

In the following we shall derive the limiting model obtained from (1.1) as $\delta \rightarrow 0$ when imposing suitable assumptions on the function $h_{u, \delta}$ defining the boundary values of the potential (see (2.2) below) and on the permittivity $\sigma_{\delta}$ (see (2.1) below), so that information on the dielectric heterogeneity is inherited. As for the permittivity we assume that it is constant (normalized to 1) in $\Omega(u)$ and a reinforced limit $\sigma_{\delta}=O(\delta)$ in $\mathcal{R}_{\delta}$. We then shall follow [1] to compute the Gamma limit with respect to the $L_{2-}$ topology of the family of functionals $\left(G_{\delta}\right)_{\delta \in(0,1)}$ as $\delta \rightarrow 0$, which turns out to be the functional

$$
G[\vartheta]:=\frac{1}{2} \int_{\Omega(u)}\left|\nabla\left(\vartheta+h_{u}\right)\right|^{2} \mathrm{~d}(x, z)+\frac{1}{2} \int_{D}\left(\sigma\left|\vartheta+h_{u}-\mathfrak{h}_{u}\right|^{2}\right)(x,-H) \mathrm{d} x
$$

with $h_{u}$ and $\mathfrak{h}_{u}$ defined below in (2.6) and in (2.8), respectively, see Theorem 3.1. Let us emphasize here that an utmost challenging feature of the limiting problem is that $\Omega(u)$ need not be a Lipschitz set as it may have cusps when the coincidence $\operatorname{set} \mathcal{C}(u)$ is nonempty. Therefore, the usual trace theorem is not available and a meaningful definition of $G$ requires a suitable definition of a trace on $(D \backslash \mathcal{C}(u)) \times\{-H\}$ for functions in (a subset of) $H^{1}(\Omega(u))$, see Lemmas 2.1 and 2.2. Once this issue is settled, the existence of a minimizer $\chi_{u}$ of $G$ in a suitable subset of $H^{1}(\Omega(u))$ is shown by classical arguments, see Proposition 3.3. The derivation of the corresponding EulerLagrange equation offers further challenges again related to the non-smoothness of $\Omega(u)$. Indeed, a formal computation reveals that $\psi_{u}=\chi_{u}+h_{u}$ solves Laplace's 
equation on $\Omega(u)$ with a Robin boundary condition along the interface $\Sigma(u)$ and a Dirichlet condition on the other boundary parts; that is,

$$
\begin{aligned}
\Delta \psi_{u} & =0 \text { in } \Omega(u), \\
\psi_{u} & =h_{u} \quad \text { on } \partial \Omega(u) \backslash \Sigma(u), \\
-\partial_{z} \psi_{u}+\sigma\left(\psi_{u}-\mathfrak{h}_{u}\right) & =0 \text { on } \Sigma(u) .
\end{aligned}
$$

However, a rigorous computation relies on Gauß' theorem which requires some geometric condition on the boundary of $\Omega(u)$ and the existence of boundary traces for $\nabla \chi_{u}$, see [7]. ${ }^{1}$ Due to the Robin boundary condition the resulting model is consistent in the sense that touching plates again do not lead to a singularity in the equations.

Remark 1.1 Of course, if the coincidence set $\mathcal{C}(u)$ is empty, then $\Omega(u)$ is a Lipschitz domain and the derivation of (1.2) only requires that $\chi_{u}$ belongs to $H^{2}(\Omega(u))$. However, this property is not guaranteed by classical elliptic regularity theory since $\Omega(u)$ is only Lipschitz. In the special case that $D$ is a one-dimensional interval and under appropriate choices of $h_{u}$ and $\mathfrak{h}_{u}$, we provide a rigorous justification of (1.2) in Theorem 3.5.

In Sect. 2 we first list the precise assumptions that we impose on the permittivity $\sigma_{\delta}$ and on the function $h_{u, \delta}$ defining the boundary values of the electrostatic potential. Moreover, since, as pointed out above, the set $\Omega(u)$ may not be Lipschitz for deformations $u$ with non-empty coincidence set $\mathcal{C}(u)$ and thus standard trace theorems are not valid, we derive in Sect. 2 also boundary trace theorems in weighted spaces for functions in $H^{1}(\Omega(u))$. Section 3 is dedicated to the computation of the Gamma limit of $\left(G_{\delta}\right)_{\delta \in(0,1)}$ as $\delta \rightarrow 0$, which is the main result of this paper, see Theorem 3.1. Moreover, we derive in Sect. 3 the limiting equations (1.2).

From now on, the function $u$ is fixed and assumed to satisfy

$$
u \in H_{0}^{1}(D) \cap C(\bar{D}) \text { with } u \geq-H \text { in } D
$$

and

$$
\Omega(u) \text { satisfies the segment property }
$$

in the sense of [11, Definition 10.23].

\section{Assumptions and Auxiliary Results}

In this section we state the precise assumptions imposed on the permittivity $\sigma_{\delta}$ and the function $h_{u, \delta}$ defining the boundary values of the electrostatic potential. We also provide some auxiliary results regarding boundary traces for functions defined on the possibly non-Lipschitz set $\Omega(u)$.

\footnotetext{
1 We thank Elmar Schrohe for pointing out this reference.
} 


\subsection{Assumptions on $\sigma_{\delta}$ and $h_{u, \delta}$}

To inherit in the limit $\delta \rightarrow 0$ the information of the permittivity from the insulating layer, we specifically assume that the permittivity scales with the layer's thickness; that is, we assume that the permittivity of the device is given in the form

$$
\sigma_{\delta}(x, z):= \begin{cases}\delta \sigma(x, z), & (x, z) \in \mathcal{R}_{\delta}, \\ 1, & (x, z) \in \Omega(u),\end{cases}
$$

for $\delta \in(0,1)$, where $\sigma \in C(\bar{D} \times[-H-1,-H])$ is a fixed function with

$$
\sigma_{\max }:=\max _{\bar{D} \times[-H-1,-H]} \sigma, \quad \sigma_{\min }:=\min _{\bar{D} \times[-H-1,-H]} \sigma>0 .
$$

Regarding the boundary values of the electrostatic potential given in (1.1c) we fix two $C^{2}$-functions

$$
h_{b}: \bar{D} \times[-H-1,-H] \times[-H, \infty) \rightarrow \mathbb{R}
$$

and

$$
h: \bar{D} \times[-H, \infty) \times[-H, \infty) \rightarrow \mathbb{R}
$$

satisfying

$$
\begin{aligned}
h_{b}(x,-H, w) & =h(x,-H, w), \\
\sigma(x,-H) \partial_{z} h_{b}(x,-H, w) & =\partial_{z} h(x,-H, w),
\end{aligned}
$$

for $(x, w) \in D \times[-H, \infty)$. We then define

$$
h_{\delta}(x, z, w):=h_{b}\left(x,-H+\frac{z+H}{\delta}, w\right)
$$

for $(x, z, w) \in \bar{D} \times[-H-\delta,-H) \times[-H, \infty)$ and

$$
h_{\delta}(x, z, w):=h(x, z, w)
$$

for $(x, z, w) \in \bar{D} \times[-H, \infty) \times[-H, \infty)$, and observe that, by (2.2), for $(x, w) \in$ $\bar{D} \times[-H, \infty)$,

$$
\begin{aligned}
\lim _{z \searrow-H} h_{\delta}(x, z, w) & =\lim _{z \nearrow-H} h_{\delta}(x, z, w), \\
\lim _{z \searrow-H} \sigma_{\delta}(x, z) \partial_{z} h_{\delta}(x, z, w) & =\lim _{z \nearrow-H} \sigma_{\delta}(x, z) \partial_{z} h_{\delta}(x, z, w) .
\end{aligned}
$$

In the following, we shall also use the abbreviations

$$
h_{u, \delta}(x, z):=h_{\delta}(x, z, u(x)), \quad(x, z) \in \Omega_{\delta}(u),
$$


and

$$
h_{u}(x, z):=h(x, z, u(x)), \quad(x, z) \in \Omega(u) .
$$

Then (2.4) entails

$$
\llbracket h_{u, \delta} \rrbracket=\llbracket \sigma_{\delta} \partial_{z} h_{u, \delta} \rrbracket=0 \text { on } \Sigma(u) .
$$

Furthermore, we set

$$
\mathfrak{h}_{u}(x,-H):=h_{b}(x,-H-1, u(x)), \quad x \in \bar{D} .
$$

\subsection{Traces in $H^{1}(\Omega(u))$}

As pointed out already in the introduction, the region $\Omega(u)$ need not be Lipschitz (besides not being connected) when the elastic plate touches the insulating layer; that is, when $\mathcal{C}(u) \neq \emptyset$. That there is still a meaningful definition of boundary traces on $D \backslash \mathcal{C}(u)$ for functions in $H^{1}(\Omega(u))$ in this case is the content of the subsequent result. We follow [14], exploiting the special geometry of $\Omega(u)$ to show that traces are well-defined in weighted spaces.

Lemma 2.1 Suppose (1.3) and set $M_{u}:=\|H+u\|_{L_{\infty}(D)}$.

(a) There exists a bounded linear operator

$$
\gamma_{u} \in \mathcal{L}\left(H^{1}(\Omega(u)), L_{2}(D \backslash \mathcal{C}(u),(H+u) d x)\right)
$$

such that $\gamma_{u} \vartheta=\vartheta(\cdot, u)$ for $\vartheta \in C^{1}(\overline{\Omega(u)})$ and

$$
\begin{aligned}
& \int_{D \backslash \mathcal{C}(u)}\left|\gamma_{u} \vartheta\right|^{2}(H+u) \mathrm{d} x \\
& \leq\|\vartheta\|_{L_{2}(\Omega(u))}^{2}+2 M_{u}\|\vartheta\|_{L_{2}(\Omega(u))}\left\|\partial_{z} \vartheta\right\|_{L_{2}(\Omega(u))}
\end{aligned}
$$

(b) There exists a bounded linear operator

$$
\gamma_{b} \in \mathcal{L}\left(H^{1}(\Omega(u)), L_{2}(D \backslash \mathcal{C}(u),(H+u) d x)\right)
$$

such that $\gamma_{b} \vartheta=\vartheta(\cdot,-H)$ for $\vartheta \in C^{1}(\overline{\Omega(u)})$ and

$$
\begin{aligned}
& \int_{D \backslash \mathcal{C}(u)}\left|\gamma_{b} \vartheta\right|^{2}(H+u) \mathrm{d} x \\
& \leq\|\vartheta\|_{L_{2}(\Omega(u))}^{2}+2 M_{u}\|\vartheta\|_{L_{2}(\Omega(u))}\left\|\partial_{z} \vartheta\right\|_{L_{2}(\Omega(u))}
\end{aligned}
$$

Proof (a) Let $\vartheta \in C^{1}(\overline{\Omega(u)})$. For $x \notin \mathcal{C}(u)$ and $z \in(-H, u(x))$, it follows from Hölder's inequality that 


$$
\begin{aligned}
\vartheta(x, u(x))^{2} & =\vartheta(x, z)^{2}+2 \int_{z}^{u(x)} \vartheta\left(x, z_{*}\right) \partial_{z} \vartheta\left(x, z_{*}\right) \mathrm{d} z_{*} \\
& \leq \vartheta(x, z)^{2}+2\left(\int_{-H}^{u(x)}\left|\vartheta\left(x, z_{*}\right)\right|^{2} \mathrm{~d} z_{*}\right)^{1 / 2}\left(\int_{-H}^{u(x)}\left|\partial_{z} \vartheta\left(x, z_{*}\right)\right|^{2} \mathrm{~d} z_{*}\right)^{1 / 2} .
\end{aligned}
$$

Hence,

$$
\begin{aligned}
(H & +u)(x) \vartheta(x, u(x))^{2} \\
& \leq \int_{-H}^{u(x)} \vartheta(x, z)^{2} \mathrm{~d} z \\
& +2(H+u)(x)\left(\int_{-H}^{u(x)}\left|\vartheta\left(x, z_{*}\right)\right|^{2} \mathrm{~d} z_{*}\right)^{1 / 2}\left(\int_{-H}^{u(x)}\left|\partial_{z} \vartheta\left(x, z_{*}\right)\right|^{2} \mathrm{~d} z_{*}\right)^{1 / 2} .
\end{aligned}
$$

We use once more Hölder's inequality to obtain

$$
\begin{aligned}
& \int_{D \backslash \mathcal{C}(u)}\left|\gamma_{b} \vartheta\right|^{2}(H+u) \mathrm{d} x \\
& \leq\|\vartheta\|_{L_{2}(\Omega(u))}^{2}+2 M_{u}\|\vartheta\|_{L_{2}(\Omega(u))}\left\|\partial_{z} \vartheta\right\|_{L_{2}(\Omega(u))}
\end{aligned}
$$

Owing to $(1.3 \mathrm{~b})$, the space $C^{1}(\overline{\Omega(u)})$ is dense in $H^{1}(\Omega(u))$ according to [11, Theorem 10.29] or [15, II.Theorem 3.1]. We then infer from (2.11) that the mapping $\vartheta \mapsto \vartheta(\cdot, u)$ from $C^{1}(\overline{\Omega(u)})$ to $L_{2}(D \backslash \mathcal{C}(u),(H+u) \mathrm{d} x)$ extends by density to a linear bounded operator $\gamma_{u}$ from $H^{1}(\Omega(u))$ to $L_{2}(D \backslash \mathcal{C}(u),(H+u) \mathrm{d} x)$ and which satisfies (2.11).

(b) The proof being similar to that of (a), we omit it here.

For simplicity, we use the notation

$$
\vartheta(x, u):=\gamma_{u} \vartheta(x), \quad \vartheta(x,-H):=\gamma_{b} \vartheta(x), \quad x \in D \backslash \mathcal{C}(u)
$$

for $\vartheta \in H^{1}(\Omega(u))$.

Next, we introduce $H_{B}^{1}(\Omega(u))$ as the closure in $H^{1}(\Omega(u))$ of the set

$$
\begin{aligned}
C_{B}^{1}(\overline{\Omega(u)}):=\left\{\vartheta \in C^{1}(\overline{\Omega(u)}): \vartheta(x, u(x))=0, x \in D\right. \\
\text { and } \vartheta(x, z)=0,(x, z) \in \partial D \times(-H, 0]\} .
\end{aligned}
$$

Next, we introduce $H_{B}^{1}(\Omega(u))$ as the closure in $H^{1}(\Omega(u))$ of the set

$$
\begin{aligned}
C_{B}^{1}(\overline{\Omega(u)}):= & \left\{\vartheta \in C^{1}(\overline{\Omega(u)}) \vartheta(x, u(x))=0, x \in D\right. \\
& \text { and } \vartheta(x, z)=0,(x, z) \in \partial D \times(-H, 0]\} .
\end{aligned}
$$


Since $\vartheta(x, u(x))=\vartheta(x,-H)=0$ for $x \in \mathcal{C}(u)$ and $\vartheta \in C_{B}^{1}(\overline{\Omega(u)})$, we agree upon setting $\vartheta(x, u(x))=\vartheta(x,-H):=0$ for all $x \in \mathcal{C}(u)$ and $\vartheta \in H_{B}^{1}(\Omega(u))$ in the reminder of this paper. For functions in $H_{B}^{1}(\Omega(u))$ we derive a Poincaré inequality and improve the information on the trace along $\Sigma$ from Lemma 2.1:

Lemma 2.2 Suppose (1.3) and let $\vartheta \in H_{B}^{1}(\Omega(u))$. Then

$$
\|\vartheta\|_{L_{2}(\Omega(u))} \leq 2\|H+u\|_{L_{\infty}(D)}\left\|\partial_{z} \vartheta\right\|_{L_{2}(\Omega(u))},
$$

and the trace $\vartheta \mapsto \vartheta(\cdot,-H)$ yields a bounded linear operator from $H_{B}^{1}(\Omega(u))$ to $L_{2}(D)$ with

$$
\|\vartheta(\cdot,-H)\|_{L_{2}(D)}^{2} \leq 2\|\vartheta\|_{L_{2}(\Omega(u))}\left\|\partial_{z} \vartheta\right\|_{L_{2}(\Omega(u))} .
$$

Proof Consider first $\vartheta \in C_{B}^{1}(\overline{\Omega(u)})$. Since $\vartheta(x, u(x))=0$ for $x \in D$, it follows from Hölder's inequality that, for $x \notin \mathcal{C}(u)$ and $z \in(-H, u(x))$,

$$
\begin{aligned}
|\vartheta(x, z)|^{2} & =|\vartheta(x, u(x))|^{2}-2 \int_{z}^{u(x)} \vartheta\left(x, z_{*}\right) \partial_{z} \vartheta\left(x, z_{*}\right) \mathrm{d} z_{*} \\
& \leq 2\left(\int_{-H}^{u(x)}\left|\vartheta\left(x, z_{*}\right)\right|^{2} \mathrm{~d} z_{*}\right)^{1 / 2}\left(\int_{-H}^{u(x)}\left|\partial_{z} \vartheta\left(x, z_{*}\right)\right|^{2} \mathrm{~d} z_{*}\right)^{1 / 2}
\end{aligned}
$$

Consequently, using again Hölder's inequality gives

$$
\begin{aligned}
& \|\vartheta\|_{L_{2}(\Omega(u))}^{2}=\int_{D \backslash \mathcal{C}(u)} \int_{-H}^{u(x)} \vartheta(x, z)^{2} \mathrm{~d} z \mathrm{~d} x \\
& \leq 2 \int_{D \backslash \mathcal{C}(u)}(H+u)(x)\left(\int_{-H}^{u(x)}\left|\vartheta\left(x, z_{*}\right)\right|^{2} \mathrm{~d} z_{*}\right)^{1 / 2} \\
& \quad \times\left(\int_{-H}^{u(x)}\left|\partial_{z} \vartheta\left(x, z_{*}\right)\right|^{2} \mathrm{~d} z_{*}\right)^{1 / 2} \mathrm{~d} x \\
& \leq 2\|H+u\|_{L_{\infty}(D)}\|\vartheta\|_{L_{2}(\Omega(u))}\left\|\partial_{z} \vartheta\right\|_{L_{2}(\Omega(u))} .
\end{aligned}
$$

Hence,

$$
\|\vartheta\|_{L_{2}(\Omega(u))} \leq 2\|H+u\|_{L_{\infty}(D)}\left\|\partial_{z} \vartheta\right\|_{L_{2}(\Omega(u))},
$$

and we complete the proof of (2.12) by a density argument.

Next, consider again $\vartheta \in C_{B}^{1}(\overline{\Omega(u)})$ and $x \notin \mathcal{C}(u)$. We infer from (2.14) with $z=-H$ that

$$
\vartheta(x,-H)^{2} \leq 2\left(\int_{-H}^{u(x)}\left|\vartheta\left(x, z_{*}\right)\right|^{2} \mathrm{~d} z_{*}\right)^{1 / 2}\left(\int_{-H}^{u(x)}\left|\partial_{z} \vartheta\left(x, z_{*}\right)\right|^{2} \mathrm{~d} z_{*}\right)^{1 / 2}
$$


Since $\vartheta(x,-H)=0$ for $x \in \mathcal{C}(u)$, we use once more Hölder's inequality to obtain

$$
\begin{aligned}
\int_{D} \vartheta(x,-H)^{2} \mathrm{~d} x & \leq 2 \int_{D}\left(\int_{-H}^{u(x)}\left|\vartheta\left(x, z_{*}\right)\right|^{2} \mathrm{~d} z_{*}\right)^{1 / 2}\left(\int_{-H}^{u(x)}\left|\partial_{z} \vartheta\left(x, z_{*}\right)\right|^{2} \mathrm{~d} z_{*}\right)^{1 / 2} \mathrm{~d} x \\
& \leq 2\|\vartheta\|_{L_{2}(\Omega(u))}\left\|\partial_{z} \vartheta\right\|_{L_{2}(\Omega(u))},
\end{aligned}
$$

which shows (2.13) for $\vartheta \in C_{B}^{1}(\overline{\Omega(u)})$. We again complete the proof by a density argument.

\section{The Reinforced Limit}

As announced in the introduction we shall derive the limiting equations of (1.1) as $\delta \rightarrow 0$ when assuming the reinforced limit (2.1) on the permittivity. For this we first compute the Gamma limit of the functionals $\left(G_{\delta}\right)_{\delta \in(0,1)}$ and then study the behavior of the corresponding minimizers.

\subsection{The Gamma Limit of the Electrostatic Energy}

Fix $M \geq\|u\|_{L_{\infty}(D)}+H$, so that

$$
-H \leq u(x) \leq M-H, \quad x \in D,
$$

and set

$$
\Omega_{M}:=D \times(-H-1, M) .
$$

Define for $\delta \in(0,1)$

$$
G_{\delta}[\vartheta]:= \begin{cases}\frac{1}{2} \int_{\Omega_{\delta}(u)} \sigma_{\delta}\left|\nabla\left(\vartheta+h_{u, \delta}\right)\right|^{2} \mathrm{~d}(x, z), & \vartheta \in H_{0}^{1}\left(\Omega_{\delta}(u)\right), \\ \infty, & \vartheta \in L_{2}\left(\Omega_{M}\right) \backslash H_{0}^{1}\left(\Omega_{\delta}(u)\right),\end{cases}
$$

with $h_{u, \delta}$ given in (2.5). Also, for $\vartheta \in H_{B}^{1}(\Omega(u))$, we set

$$
G[\vartheta]:=\frac{1}{2} \int_{\Omega(u)}\left|\nabla\left(\vartheta+h_{u}\right)\right|^{2} \mathrm{~d}(x, z)+\frac{1}{2} \int_{D}\left(\sigma\left|\vartheta+h_{u}-\mathfrak{h}_{u}\right|^{2}\right)(x,-H) \mathrm{d} x,
$$

with $h_{u}$ and $\mathfrak{h}_{u}$ defined in (2.6) and (2.8), respectively, and

$$
G[\vartheta]:=\infty, \quad \vartheta \in L_{2}\left(\Omega_{M}\right) \backslash H_{B}^{1}(\Omega(u)) .
$$

Then the main result of the present paper is the following convergence. 
Theorem 3.1 Suppose (1.3) and (2.2)-(2.3). Then

$$
\Gamma-\lim _{\delta \rightarrow 0} G_{\delta}=G \text { in } L_{2}\left(\Omega_{M}\right)
$$

For more information on Gamma convergence we refer, e.g., to [5].

Proof (i) Asymptotic weak lower semi-continuity. Considering

$$
\vartheta_{\delta} \rightarrow \vartheta_{0} \text { in } L_{2}\left(\Omega_{M}\right)
$$

we shall show that

$$
G\left[\vartheta_{0}\right] \leq \liminf _{\delta \rightarrow 0} G_{\delta}\left[\vartheta_{\delta}\right]
$$

Due to the definitions of the functionals we may assume without loss of generality that $\vartheta_{\delta} \in H_{0}^{1}\left(\Omega_{\delta}(u)\right)$ for $\delta \in(0,1)$ and

$$
\sup _{\delta \in(0,1)} G_{\delta}\left[\vartheta_{\delta}\right]<\infty
$$

Therefore, by (2.1), (2.2), and (3.4), we have

$$
\sup _{\delta \in(0,1)}\left\|\nabla \vartheta_{\delta}\right\|_{L_{2}(\Omega(u))}<\infty
$$

Thus, invoking (3.3) and (3.5) we may further assume that

$$
\vartheta_{\delta} \rightarrow \vartheta_{0} \text { in } H^{1}(\Omega(u))
$$

Since $\vartheta_{\delta}$ belongs to $H_{0}^{1}\left(\Omega_{\delta}(u)\right)$, which is the closure of $C_{c}^{\infty}\left(\Omega_{\delta}(u)\right)$ in $H^{1}\left(\Omega_{\delta}(u)\right)$, and $C_{c}^{\infty}\left(\Omega_{\delta}(u)\right) \subset C_{B}^{1}(\Omega(u))$, it readily follows from the definitions of $\Omega_{\delta}(u)$ and $\Omega(u)$ that $\vartheta_{\delta}$ belongs to $H_{B}^{1}(\Omega(u))$, the latter being a closed subspace of $H^{1}(\Omega(u))$. Thus (3.6) implies that $\vartheta_{0} \in H_{B}^{1}(\Omega(u)$ ). Moreover, (2.13), (3.3), and (3.5) yield

$$
\vartheta_{\delta}(\cdot,-H) \rightarrow \vartheta_{0}(\cdot,-H) \text { in } L_{2}(D)
$$

Next, since, for each $\varepsilon>0$, there is $\delta_{\varepsilon} \in(0,1)$ such that

$$
|\sigma(x, z)-\sigma(x,-H)| \leq \varepsilon, \quad(x, z) \in \mathcal{R}_{\delta_{\varepsilon}},
$$


it follows from (3.4) that

$$
\begin{aligned}
& \liminf _{\delta \rightarrow 0} \delta \int_{\mathcal{R}_{\delta}} \sigma(x, z)\left|\nabla\left(\vartheta_{\delta}+h_{u, \delta}\right)\right|^{2} \mathrm{~d}(x, z) \\
& =\liminf _{\delta \rightarrow 0} \delta \int_{\mathcal{R}_{\delta}} \sigma(x,-H)\left|\nabla\left(\vartheta_{\delta}+h_{u, \delta}\right)\right|^{2} \mathrm{~d}(x, z) \\
& \geq \liminf _{\delta \rightarrow 0} \delta \int_{\mathcal{R}_{\delta}} \sigma(x,-H)\left|\partial_{z}\left(\vartheta_{\delta}+h_{u, \delta}\right)\right|^{2} \mathrm{~d}(x, z) .
\end{aligned}
$$

The property $\vartheta_{\delta}(\cdot,-H-\delta)=0$ a.e. in $D$ and Hölder's inequality yield

$$
\left|\left(\vartheta_{\delta}+h_{u, \delta}\right)(x,-H)-h_{u, \delta}(x,-H-\delta)\right|^{2} \leq \delta \int_{-H-\delta}^{-H}\left|\partial_{z}\left(\vartheta_{\delta}+h_{u, \delta}\right)(x, z)\right|^{2} \mathrm{~d} z
$$

for a.e. $x \in D$ while (2.2c) and (2.3) imply for $x \in D$ (recalling (2.6) and (2.8))

$$
h_{u, \delta}(x,-H)=h_{u}(x,-H), \quad h_{u, \delta}(x,-H-\delta)=\mathfrak{h}_{u}(x,-H) .
$$

Consequently,

$$
\begin{aligned}
& \liminf _{\delta \rightarrow 0} \delta \int_{\mathcal{R}_{\delta}} \sigma(x, z)\left|\nabla\left(\vartheta_{\delta}+h_{u, \delta}\right)\right|^{2} \mathrm{~d}(x, z) \\
& \geq \liminf _{\delta \rightarrow 0} \int_{D} \sigma(x,-H)\left|\vartheta_{\delta}(x,-H)+h_{u}(x,-H)-\mathfrak{h}_{u}(x,-H)\right|^{2} \mathrm{~d} x \\
& =\int_{D} \sigma(x,-H)\left|\vartheta_{0}(x,-H)+h_{u}(x,-H)-\mathfrak{h}_{u}(x,-H)\right|^{2} \mathrm{~d} x,
\end{aligned}
$$

where we used (3.7) and $\vartheta_{\delta}(x,-H)=0, x \in \mathcal{C}(u)$ (since $\left.\vartheta_{\delta} \in H_{0}^{1}\left(\Omega_{\delta}(u)\right)\right)$ to derive the last equality. Since $h_{u, \delta}=h_{u}$ and $\sigma_{\delta}=1$ in $\Omega(u)$ it follows from (3.6) that

$$
\frac{1}{2} \int_{\Omega(u)}\left|\nabla\left(\vartheta_{0}+h_{u}\right)\right|^{2} \mathrm{~d}(x, z) \leq \liminf _{\delta \rightarrow 0} \frac{1}{2} \int_{\Omega(u)} \sigma_{\delta}\left|\nabla\left(\vartheta_{\delta}+h_{u, \delta}\right)\right|^{2} \mathrm{~d}(x, z) .
$$

Therefore, gathering the last two inequalities gives

$$
\liminf _{\delta \rightarrow 0} G_{\delta}\left[\vartheta_{\delta}\right] \geq G\left[\vartheta_{0}\right]
$$

and thus the weak lower semi-continuity of the functionals $\left(G_{\delta}\right)_{\delta \in(0,1)}$ follows.

(ii) Recovery sequence. To prove the existence of a recovery sequence it suffices, by definition of the functionals $\left(G_{\delta}\right)_{\delta \in(0,1)}$, to consider $\vartheta \in H_{B}^{1}(\Omega(u))$. Let $\bar{\vartheta}$ denote the 
trivial extension of $\vartheta$ to $D \times(-H, M)$ and then its reflection to $D \times(-2 H-M, M)$; that is,

$$
\bar{\vartheta}(x, z):= \begin{cases}0, & x \in D, u(x)<z<M \\ \vartheta(x, z), & x \in D,-H<z \leq u(x) \\ \vartheta(x,-2 H-z), & x \in D,-2 H-u(x)<z \leq-H \\ 0, & x \in D,-2 H-M<z \leq-2 H-u(x) .\end{cases}
$$

Let

$$
\tau_{\delta}(x):=\left\{\begin{array}{ll}
1, & d(x, \partial D)>\sqrt{\delta}, \\
\frac{d(x, \partial D)}{\sqrt{\delta}}, & d(x, \partial D) \leq \sqrt{\delta},
\end{array} \quad x \in D,\right.
$$

where $d(\cdot, \partial D)$ denotes the distance to $\partial D$. Since the $C^{2}$-regularity of the boundary of $D$ implies that $d(\cdot, \partial D)$ is $C^{2}$ near $\partial D$ (see [6, Lemma 14.16]), we have $\tau_{\delta} \in H^{1}(D)$ for $\delta$ small enough. Define now

$$
\begin{aligned}
\vartheta_{\delta}(x, z):= & \frac{z+H+\delta}{\delta} \bar{\vartheta}(x, z)+\frac{z+H+\delta}{\delta}\left[h_{u, \delta}(x,-H)-h_{u, \delta}(x,-H-\delta)\right] \tau_{\delta}(x) \\
& -\left[h_{u, \delta}(x, z)-h_{u, \delta}(x,-H-\delta)\right] \tau_{\delta}(x), \quad(x, z) \in \mathcal{R}_{\delta},
\end{aligned}
$$

and

$$
\vartheta_{\delta}(x, z):=\vartheta(x, z), \quad(x, z) \in \Omega(u) .
$$

The regularities of $\vartheta, \bar{\vartheta}$, and $\tau_{\delta}$ imply that $\vartheta_{\delta} \in H^{1}\left(\mathcal{R}_{\delta}\right) \cap H^{1}(\Omega(u))$ and thus, since moreover $\llbracket \vartheta_{\delta} \rrbracket=0$ on $\Sigma(u)$, we deduce $\vartheta_{\delta} \in H^{1}\left(\Omega_{\delta}(u)\right)$. By construction, it follows that $\vartheta_{\delta}$ vanishes on $\partial \Omega_{\delta}(u)$, hence $\vartheta_{\delta} \in H_{0}^{1}\left(\Omega_{\delta}(u)\right)$. We now claim that

$$
G[\vartheta]=\lim _{\delta \rightarrow 0} G_{\delta}\left[\vartheta_{\delta}\right]
$$

Indeed, for $(x, z) \in \mathcal{R}_{\delta}$ we note that

$$
\begin{aligned}
\partial_{z}\left(\vartheta_{\delta}+h_{u, \delta}\right)(x, z) \\
=\frac{1}{\delta} \bar{\vartheta}(x, z)+\frac{1}{\delta}\left[h_{u, \delta}(x,-H)-h_{u, \delta}(x,-H-\delta)\right] \tau_{\delta}(x) \\
\quad+\frac{z+H+\delta}{\delta} \partial_{z} \bar{\vartheta}(x, z)+\left(1-\tau_{\delta}(x)\right) \partial_{z} h_{u, \delta}(x, z)
\end{aligned}
$$

and then handle the terms separately. From (2.3) and $\sigma_{\delta}=\delta \sigma$ in $\mathcal{R}_{\delta}$ we obtain 


$$
\begin{aligned}
& \int_{\mathcal{R}_{\delta}} \sigma_{\delta}(x, z)\left|\frac{1}{\delta} \bar{\vartheta}(x, z)+\frac{1}{\delta}\left[h_{u, \delta}(x,-H)-h_{u, \delta}(x,-H-\delta)\right] \tau_{\delta}(x)\right|^{2} \mathrm{~d}(x, z) \\
& =\frac{1}{\delta} \int_{-H-\delta}^{-H} \int_{D} \sigma(x, z)\left|\bar{\vartheta}(x, z)+\left[h_{u}(x,-H)-\mathfrak{h}_{u}(x,-H)\right] \tau_{\delta}(x)\right|^{2} \mathrm{~d} x \mathrm{~d} z .
\end{aligned}
$$

Thus, recalling the definition of $\tau_{\delta}$ and using Lebesgue's theorem,

$$
\begin{aligned}
& \lim _{\delta \rightarrow 0} \int_{\mathcal{R}_{\delta}} \sigma_{\delta}(x, z)\left|\frac{1}{\delta} \bar{\vartheta}(x, z)+\frac{1}{\delta}\left[h_{u, \delta}(x,-H)-h_{u, \delta}(x,-H-\delta)\right] \tau_{\delta}(x)\right|^{2} \mathrm{~d}(x, z) \\
& =\int_{D} \sigma(x,-H)\left|\bar{\vartheta}(x,-H)+h_{u}(x,-H)-\mathfrak{h}_{u}(x,-H)\right|^{2} \mathrm{~d} x .
\end{aligned}
$$

Next, we have

$$
\int_{\mathcal{R}_{\delta}} \sigma_{\delta}(x, z)\left|\frac{z+H+\delta}{\delta} \partial_{z} \bar{\vartheta}(x, z)\right|^{2} \mathrm{~d}(x, z) \leq \delta \sigma_{\max } \int_{-H-\delta}^{-H} \int_{D}\left|\partial_{z} \bar{\vartheta}(x, z)\right|^{2} \mathrm{~d} x \mathrm{~d} z,
$$

so that

$$
\lim _{\delta \rightarrow 0} \int_{\mathcal{R}_{\delta}} \sigma_{\delta}(x, z)\left|\frac{z+H+\delta}{\delta} \partial_{z} \bar{\vartheta}(x, z)\right|^{2} \mathrm{~d}(x, z)=0
$$

since $\bar{\vartheta} \in H^{1}(D \times(-2 H-M, M))$. Moreover, from (2.3) it follows that

$$
\partial_{z} h_{u, \delta}(x, z)=\frac{1}{\delta} \partial_{z} h_{1}\left(x,-H+\frac{z+H}{\delta}, u(x)\right), \quad(x, z) \in \mathcal{R}_{\delta},
$$

from which we get, using substitution,

$$
\begin{aligned}
& \int_{\mathcal{R}_{\delta}} \sigma_{\delta}(x, z)\left|\left(1-\tau_{\delta}(x)\right) \partial_{z} h_{u, \delta}(x, z)\right|^{2} \mathrm{~d}(x, z) \\
& \quad \leq \sigma_{\max } \int_{-H-1}^{-H} \int_{D}\left|\left(1-\tau_{\delta}(x)\right) \partial_{z} h_{1}(x, \xi, u(x))\right|^{2} \mathrm{~d} x \mathrm{~d} \xi
\end{aligned}
$$

Hence, by definition of $\tau_{\delta}$ and Lebesgue's theorem,

$$
\lim _{\delta \rightarrow 0} \int_{\mathcal{R}_{\delta}} \sigma_{\delta}(x, z)\left|\left(1-\tau_{\delta}(x)\right) \partial_{z} h_{u, \delta}(x, z)\right|^{2} \mathrm{~d}(x, z)=0 .
$$

Gathering (3.9), (3.10), (3.11), and (3.13) we derive

$$
\begin{aligned}
\lim _{\delta} \rightarrow 0 & \int_{\mathcal{R}_{\delta}} \sigma_{\delta}(x, z)\left|\partial_{z}\left(\vartheta_{\delta}+h_{u, \delta}\right)\right|^{2} \mathrm{~d}(x, z) \\
& =\int_{D} \sigma(x,-H)\left|\vartheta(x,-H)+h_{u}(x,-H)-\mathfrak{h}_{u}(x,-H)\right|^{2} \mathrm{~d} x .
\end{aligned}
$$


Next, still for $(x, z) \in \mathcal{R}_{\delta}$, we compute

$$
\begin{aligned}
\nabla_{x} \vartheta_{\delta}(x, z)= & \frac{z+H+\delta}{\delta} \nabla_{x} \bar{\vartheta}(x, z) \\
& +\frac{z+H+\delta}{\delta}\left[\nabla_{x} h_{u, \delta}(x,-H)-\nabla_{x} h_{u, \delta}(x,-H-\delta)\right] \tau_{\delta}(x) \\
& +\frac{z+H+\delta}{\delta}\left[h_{u, \delta}(x,-H)-h_{u, \delta}(x,-H-\delta)\right] \nabla_{x} \tau_{\delta}(x) \\
& -\left[\nabla_{x} h_{u, \delta}(x, z)-\nabla_{x} h_{u, \delta}(x,-H-\delta)\right] \tau_{\delta}(x) \\
& -\left[h_{u, \delta}(x, z)-h_{u, \delta}(x,-H-\delta)\right] \nabla_{x} \tau_{\delta}(x),
\end{aligned}
$$

where

$$
\begin{aligned}
\nabla_{x} h_{u, \delta}(x, z)= & \nabla_{x} h_{1}\left(x,-H+\frac{z+H}{\delta}, u(x)\right) \\
& +\partial_{w} h_{b}\left(x,-H+\frac{z+H}{\delta}, u(x)\right) \nabla_{x} u(x)
\end{aligned}
$$

We further note that

$$
0 \leq \tau_{\delta}(x) \leq 1, \quad 0 \leq \frac{z+H+\delta}{\delta} \leq 1
$$

for $(x, z) \in \mathcal{R}_{\delta}$. Gathering these observations, recalling that $\sigma_{\delta}=\delta \sigma$ in $\mathcal{R}_{\delta}$, and denoting the norm of $h_{b}$ in $C^{1}(\bar{D} \times[-H-1,-H] \times[-H, M])$ by $\left\|h_{b}\right\|_{C^{1}}$ we deduce

$$
\begin{aligned}
& \int_{\mathcal{R}_{\delta}} \sigma_{\delta}(x, z)\left|\nabla_{x}\left(\vartheta_{\delta}+h_{u, \delta}\right)\right|^{2} \mathrm{~d}(x, z) \\
& \leq c \delta \sigma_{\max } \int_{\mathcal{R}_{\delta}}\left|\nabla_{x} \bar{\vartheta}(x, z)\right|^{2} \mathrm{~d}(x, z) \\
& \quad+c \delta \sigma_{\max }\left\|h_{b}\right\|_{C^{1}}^{2} \int_{-H-\delta}^{-H} \int_{D}\left(1+\left|\nabla_{x} u(x)\right|^{2}+\left|\nabla_{x} \tau_{\delta}(x)\right|^{2}\right) \mathrm{d} x \mathrm{~d} z \\
& \leq c \delta \sigma_{\max } \int_{\mathcal{R}_{\delta}}\left|\nabla_{x} \bar{\vartheta}(x, z)\right|^{2} \mathrm{~d}(x, z) \\
& \quad+c \delta^{2} \sigma_{\max }\left\|h_{b}\right\|_{C^{1}}^{2} \int_{D}\left(1+\left|\nabla_{x} u(x)\right|^{2}+\left|\nabla_{x} \tau_{\delta}(x)\right|^{2}\right) \mathrm{d} x
\end{aligned}
$$

Now, since the distance function $d(\cdot, \partial D) \in C^{2}$ (see [6, Lemma 14.16]) satisfies the eikonal equation we have

$$
\left|\nabla_{x} \tau_{\delta}(x)\right| \leq \frac{1}{\sqrt{\delta}}, \quad x \in D
$$


and since $u \in H_{0}^{1}(D)$ and $\bar{\vartheta} \in H^{1}(D \times(-2 H-M, M))$, we deduce that the righthand side of the above estimate is of order $\delta$, hence

$$
\lim _{\delta \rightarrow 0} \int_{\mathcal{R}_{\delta}} \sigma_{\delta}(x, z)\left|\nabla_{x}\left(\vartheta_{\delta}+h_{u, \delta}\right)\right|^{2} \mathrm{~d}(x, z)=0 .
$$

Consequently, we derive from (2.1a), (2.3), (3.14), (3.16), and (2.2c)

$$
\begin{aligned}
\lim _{\delta \rightarrow 0} & \int_{\Omega_{\delta}(u)} \sigma_{\delta}\left|\nabla\left(\vartheta_{\delta}+h_{u, \delta}\right)\right|^{2} \mathrm{~d}(x, z) \\
= & \int_{\Omega(u)}\left|\nabla\left(\vartheta+h_{u}\right)\right|^{2} \mathrm{~d}(x, z) \\
& +\int_{D} \sigma(x,-H)\left|\vartheta(x,-H)+h_{u}(x,-H)-\mathfrak{h}_{u}(x,-H)\right|^{2} \mathrm{~d} x \\
= & 2 G[\vartheta]
\end{aligned}
$$

that is, (3.8) since $\vartheta_{\delta} \in H_{0}^{1}\left(\Omega_{\delta}(u)\right)$. This proves the assertion.

\subsection{Minimizers}

Now that we have shown the Gamma convergence of the functionals $\left(G_{\delta}\right)_{\delta \in(0,1)}$ towards $G$, we can deduce useful information on the relation between their minimizers. We first recall from the Lax-Milgram theorem (also see [10, Proposition 3.1, Lemma 3.2]) the following result regarding the solvability of the transmission problem (1.1):

Proposition 3.2 Suppose (1.3) and (2.2)-(2.3). For $\delta \in(0,1)$, there is a unique minimizer $\chi_{u, \delta} \in H_{0}^{1}\left(\Omega_{\delta}(u)\right)$ of the functional $G_{\delta}$ on $H_{0}^{1}\left(\Omega_{\delta}(u)\right)$. It satisfies

$$
\int_{\Omega_{\delta}(u)} \sigma_{\delta}\left|\nabla \chi_{u, \delta}\right|^{2} d(x, z) \leq 4 \int_{\Omega_{\delta}(u)} \sigma_{\delta}\left|\nabla h_{u, \delta}\right|^{2} d(x, z) .
$$

In addition, $\psi_{u, \delta}:=\chi_{u, \delta}+h_{u, \delta}$ is a variational solution to (1.1).

As for the functional $G$ we note:

Proposition 3.3 Suppose (1.3) and (2.2). There is a unique minimizer $\chi_{u} \in H_{B}^{1}(\Omega(u))$ of the functional $G$ on $H_{B}^{1}(\Omega(u))$. It satisfies

$$
\begin{aligned}
\left\|\nabla \chi_{u}\right\|_{L_{2}(\Omega(u))}^{2} & +\left\|\sqrt{\sigma} \chi_{u}(\cdot,-H)\right\|_{L_{2}(D)}^{2} \\
& \leq 4\left\|\nabla h_{u}\right\|_{L_{2}(\Omega(u))}^{2}+4\left\|\sqrt{\sigma}\left(h_{u}-\mathfrak{h}_{u}\right)(\cdot,-H)\right\|_{L_{2}(D)}^{2} .
\end{aligned}
$$

Proof It readily follows from (2.1b), the Poincaré inequality (2.12), and the LaxMilgram theorem that there is a unique minimizer $\chi_{u} \in H_{B}^{1}(\Omega(u))$ of $G$ on $H_{B}^{1}(\Omega(u))$. Since $\chi_{u}$ satisfies

$$
G\left[\chi_{u}\right] \leq G[\vartheta], \quad \vartheta \in H_{B}^{1}(\Omega(u)),
$$


we obtain the claimed estimate by taking $\vartheta \equiv 0$ in the previous inequality.

As a consequence of Theorem 3.1 and Propositions 3.2 and 3.3, we obtain the convergence of the minimizers of the functionals.

Corollary 3.4 Suppose (1.3) and (2.2)-(2.3). Then

$$
\chi_{u, \delta} \longrightarrow \chi_{u} \quad \text { in } L_{2}(\Omega(u)) \quad \text { and } \quad \chi_{u, \delta} \rightarrow \chi_{u} \quad \text { in } H^{1}(\Omega(u))
$$

as $\delta \rightarrow 0$, and

$$
\lim _{\delta \rightarrow 0} G_{\delta}\left[\chi_{u, \delta}\right]=G\left[\chi_{u}\right]
$$

Proof Let $\delta \in(0,1)$. We use (2.1a), (3.12), and (3.15) to obtain that

$$
\int_{\Omega_{\delta}(u)} \sigma_{\delta}\left|\nabla h_{u, \delta}\right|^{2} \mathrm{~d}(x, z) \leq c_{1}
$$

for a constant $c_{1}>0$ independent of $\delta$. This estimate, along with (2.1b) and (3.17), implies that

$$
\left\|\nabla \chi_{u, \delta}\right\|_{L_{2}(\Omega(u))}^{2}+\delta\left\|\nabla \chi_{u, \delta}\right\|_{L_{2}\left(\mathcal{R}_{\delta}\right)}^{2} \leq c_{1} .
$$

On the one hand, we infer from the Poincaré inequality (2.12) and (3.18) that

$$
\left\|\chi_{u, \delta}\right\|_{H^{1}(\Omega(u))} \leq c_{2} .
$$

On the other hand, since $\chi_{u, \delta}(\cdot,-H-\delta) \equiv 0$ we can use the same argument as for the derivation of (2.12) to show that

$$
\left\|\chi_{u, \delta}\right\|_{L_{2}\left(\mathcal{R}_{\delta}\right)} \leq 2 \delta\left\|\partial_{z} \chi_{u, \delta}\right\|_{L_{2}\left(\mathcal{R}_{\delta}\right)} .
$$

Therefore, using (3.18) and the trivial extension of $\chi_{u, \delta}$,

$$
\left\|\chi_{u, \delta}\right\|_{L_{2}(D \times(-H-1,-H))} \leq c_{3} \sqrt{\delta} .
$$

Now, despite of the possible non-Lipschitz character of $\Omega(u)$, the embedding of $H^{1}(\Omega(u))$ in $L_{2}(\Omega(u))$ is compact, see [15, I.Theorem 1.4] or [11, Theorem 11.21], and we infer from (3.19) and (3.20) that there are a function $\zeta \in L_{2}\left(\Omega_{M}\right) \cap H^{1}(\Omega(u))$ vanishing in $\Omega_{M} \backslash \Omega(u)$ and a sequence $\delta_{n} \rightarrow 0$ such that $\chi_{u, \delta_{n}} \rightarrow \zeta$ in $L_{2}\left(\Omega_{M}\right)$ and $\chi_{u, \delta_{n}} \rightarrow \zeta$ in $H^{1}(\Omega(u))$. Thus, Theorem 3.1 and the fundamental theorem of $\Gamma$ convergence [5, Corollary 7.20] imply that $\zeta$ is a minimizer of $G$ on $L_{2}\left(\Omega_{M}\right)$ and that

$$
\lim _{n \rightarrow \infty} G_{\delta_{n}}\left[\chi_{u, \delta_{n}}\right]=G[\zeta]
$$

Obviously, this implies that $\left.\zeta\right|_{\Omega(u)} \in H_{B}^{1}(\Omega(u))$ is a minimizer of $G$ on $H_{B}^{1}(\Omega(u))$, hence $\left.\zeta\right|_{\Omega(u)}=\chi_{u}$ by Proposition 3.3 and it is independent of the sequence $\left(\delta_{n}\right)_{n \in \mathbb{N}}$. 
Clearly, $G[\zeta]$ only depends on $\left.\zeta\right|_{\Omega(u)}=\chi_{u}$ and is independent of the sequence $\left(\delta_{n}\right)_{n \in \mathbb{N}}$. This proves the claim.

\subsection{The Limiting Model}

Finally, we shall derive the analogue to Eqs. (1.1) satisfied by $\psi_{u}:=\chi_{u}+h_{u}$ for which we suppose that $\chi_{u} \in H_{B}^{1}(\Omega(u)) \cap H^{2}(\Omega(u))$ and that Gauß' theorem applies for $\Omega(u)$ and $\nabla \chi_{u}$ (which requires some geometric condition on the boundary of $\Omega(u)$ and the existence of boundary traces for $\nabla \chi_{u}$, see [7]).

Let $u \in H_{0}^{1}(D) \cap C(\bar{D})$ satisfy (3.1). Since $\chi_{u} \in H_{B}^{1}(\Omega(u))$ is the minimizer of $G$ on $H_{B}^{1}(\Omega(u))$ by Corollary 3.4 , it satisfies the variational equality

$$
\begin{aligned}
0= & \int_{\Omega(u)} \nabla\left(\chi_{u}+h_{u}\right) \cdot \nabla \phi \mathrm{d}(x, z) \\
& +\int_{D} \sigma(x,-H)\left(\chi_{u}(x,-H)+h_{u}(x,-H)-\mathfrak{h}_{u}(x,-H)\right) \phi(x,-H) \mathrm{d} x
\end{aligned}
$$

for any $\phi \in H_{B}^{1}(\Omega(u))$. Then, by standard computations,

$$
\begin{aligned}
0= & \int_{\Omega(u)} \nabla\left(\chi_{u}+h_{u}\right) \cdot \nabla \phi \mathrm{d}(x, z) \\
& +\int_{D} \sigma(x,-H)\left(\chi_{u}(x,-H)+h_{u}(x,-H)-\mathfrak{h}_{u}(x,-H)\right) \phi(x,-H) \mathrm{d} x \\
= & -\int_{\Omega(u)} \Delta\left(\chi_{u}+h_{u}\right) \phi \mathrm{d}(x, z)+\int_{\partial \Omega(u)} \nabla\left(\chi_{u}+h_{u}\right) \cdot \mathbf{n}_{\partial \Omega(u)} \phi \mathrm{d} S \\
& +\int_{D} \sigma(x,-H)\left(\chi_{u}(x,-H)+h_{u}(x,-H)-\mathfrak{h}_{u}(x,-H)\right) \phi(x,-H) \mathrm{d} x .
\end{aligned}
$$

Therefore, since $\phi$ vanishes on $\partial \Omega(u) \backslash \Sigma$,

$$
\begin{aligned}
0= & -\int_{\Omega(u)} \Delta\left(\chi_{u}+h_{u}\right) \phi \mathrm{d}(x, z)-\int_{D} \partial_{z}\left(\chi_{u}+h_{u}\right)(\cdot,-H) \phi(\cdot,-H) \mathrm{d} x \\
& +\int_{D} \sigma(\cdot,-H)\left(\chi_{u}(\cdot,-H)+h_{u}(\cdot,-H)-\mathfrak{h}_{u}(\cdot,-H)\right) \phi(\cdot,-H) \mathrm{d} x .
\end{aligned}
$$

Consequently, in this case $\psi_{u}=\chi_{u}+h_{u} \in H^{2}(\Omega(u))$ solves Laplace's equation with mixed boundary conditions of Dirichlet and Robin type as announced in (1.2). The corresponding electrostatic energy is

$$
E_{e}(u):=-G\left[\psi_{u}-h_{u}\right]
$$

that is,

$$
E_{e}(u)=-\frac{1}{2} \int_{\Omega(u)}\left|\nabla \psi_{u}\right|^{2} \mathrm{~d}(x, z)
$$




$$
-\frac{1}{2} \int_{D} \sigma(x,-H)\left|\psi_{u}(x,-H)-\mathfrak{h}_{u}(x,-H)\right|^{2} \mathrm{~d} x,
$$

where $\mathfrak{h}_{u}$ is defined in (2.8). By Corollary 3.4 we have $E_{e, \delta}(u) \rightarrow E_{e}(u)$ as $\delta \rightarrow 0$.

For the special case that $D$ is an interval in $\mathbb{R}$ and $h_{b}$ is of the form

$$
h_{b}(x, z, w)=h(x,-H, w)+(z+H)(h(x,-H, w)-\mathfrak{h}(x, w))
$$

for $(x, z, w) \in \bar{D} \times[-H-1,-H] \times[-H, \infty)$ with $\mathfrak{h} \in C^{2}(\bar{D} \times[-H, \infty))$, the previous computation can be rigorously justified.

Theorem 3.5 If $D=(a, b) \subset \mathbb{R}, u \in H^{2}(D) \cap H_{0}^{1}(D)$ with $u \geq-H$ in $D$, and $h$ and $h_{b}$ satisfy (3.22), then (1.2) admits a unique solution $\psi_{u} \in H^{2}(\Omega(u))$ with $\partial_{z} \psi_{u}(\cdot,-H) \in L_{2}(D \backslash \mathcal{C}(u))$. It is given as $\psi_{u}=\chi_{u}+h_{u}$ with $\chi_{u} \in H_{B}^{1}(\Omega(u))$ being the unique minimizer of $G$ on $H_{B}^{1}(\Omega(u))$.

Remark 3.6 We point out once more that, since $\Omega(u)$ need not be Lipschitz, the stated $L_{2}$-regularity of $\partial_{z} \psi_{u}(\cdot,-H)$ does not follow from the $H^{2}$-regularity of $\psi_{u}$ by a standard trace theorem. In fact, Lemma 2.1 only ensures that $\partial_{z} \psi_{u}(\cdot,-H)$ belongs to the weighted space $L_{2}(D \backslash \mathcal{C}(u),(H+u) \mathrm{d} x)$. That $\partial_{z} \psi_{u}(\cdot,-H)$ additionally belongs to $L_{2}(D \backslash \mathcal{C}(u))$ follows a posteriori from (1.2c), as shown in the proof below.

Proof of Theorem 3.5 Since $D$ is a one-dimensional interval, $H^{2}(D)$ is embedded in $C(\bar{D})$, which implies (1.3a) as well as $(1.3 \mathrm{~b})$ by [11, Exercise 10.26]. It follows from $[3,8]$ that the unique minimizer $\chi_{u} \in H_{B}^{1}(\Omega(u))$ of $G$ on $H_{B}^{1}(\Omega(u))$ belongs to $H^{2}(\Omega(u))$. Thanks to (1.3b) and [11, Theorem 10.29], see also [15, II.Theorem 3.1], there is a sequence $\left(\chi_{u, j}\right)_{j \geq 1}$ in $C^{\infty}(\overline{\Omega(u)})$ such that

$$
\lim _{j \rightarrow \infty}\left\|\chi_{u, j}-\chi_{u}\right\|_{H^{2}(\Omega(u))}=0 .
$$

Now, for $j \geq 1$ and $\phi \in C_{B}^{1}(\overline{\Omega(u)})$, we infer from [7, Folgerung 7.5] and the regularity of $\chi_{u, j}, \phi$, and $h$ that Gauß' theorem can be applied in each connected component of $\Omega(u)$, as there are at most two singular points. Therefore, we obtain

$$
\begin{aligned}
\int_{\Omega(u)} \nabla\left(\chi_{u, j}+h_{u}\right) \cdot \nabla \phi \mathrm{d}(x, z) & \\
=- & -\int_{\Omega(u)} \Delta\left(\chi_{u, j}+h_{u}\right) \phi \mathrm{d}(x, z)+\int_{\partial \Omega(u)} \nabla\left(\chi_{u, j}+h_{u}\right) \cdot \mathbf{n}_{\partial \Omega(u)} \phi \mathrm{d} S \\
= & -\int_{\Omega(u)} \Delta\left(\chi_{u, j}+h_{u}\right) \phi \mathrm{d}(x, z)-\int_{D} \partial_{z}\left(\chi_{u, j}+h_{u}\right)(\cdot,-H) \phi(\cdot,-H) \mathrm{d} x,
\end{aligned}
$$

since $\phi$ vanishes on $\partial \Omega(u) \backslash \Sigma(u)$. Now, thanks to (3.23), it is straightforward to pass to the limit $j \rightarrow \infty$ in the two integrals over $\Omega(u)$. Moreover, by Lemma 2.1 and (3.23),

$$
\lim _{j \rightarrow \infty} \int_{D \backslash \mathcal{C}(u)}\left|\partial_{z} \chi_{u, j}(\cdot,-H)-\partial_{z} \chi_{u}(\cdot,-H)\right|^{2}(H+u) \mathrm{d} x=0
$$


Consequently, if $\phi(\cdot,-H)$ is compactly supported in $D \backslash \mathcal{C}(u)$, then

$$
\lim _{j \rightarrow \infty} \int_{D} \partial_{z}\left(\chi_{u, j}+h_{u}\right)(\cdot,-H) \phi(\cdot,-H) \mathrm{d} x=\int_{D} \partial_{z}\left(\chi_{u}+h_{u}\right)(\cdot,-H) \phi(\cdot,-H) \mathrm{d} x
$$

Thanks to the above analysis, the identity (3.21) holds true for any test function $\phi \in$ $C_{B}^{1}(\overline{\Omega(u)})$ such that $\phi(\cdot,-H)$ is compactly supported in $D \backslash \mathcal{C}(u)$. We then deduce from (3.21) that $\psi_{u}=\chi_{u}+h_{u}$ satisfies (1.2a) and (1.2b) in $L_{2}(\Omega(u))$ and $L_{2}(\partial \Omega(u) \backslash$ $\Sigma(u))$, respectively, while

$$
\partial_{z} \psi_{u}(\cdot,-H)=\left(\sigma\left(\chi_{u}+h_{u}-\mathfrak{h}_{u}\right)\right)(\cdot,-H) \text { a.e. in } D \backslash \mathcal{C}(u)
$$

Since $\chi_{u} \in H_{B}^{1}(\Omega(u))$, the right-hand side of (3.24) belongs to $L_{2}(D \backslash \mathcal{C}(u))$ by Lemma 2.2 and the regularity of $h_{u}$ and $\mathfrak{h}_{u}$, so that $\partial_{z} \psi_{u}(\cdot,-H)$ also belongs to that space.

The analysis of the complete MEMS model coupling (1.2) to an equation for $u$ is performed in a forthcoming research [8] for $n=1$.

Acknowledgements Open Access funding provided by Projekt DEAL.

Open Access This article is licensed under a Creative Commons Attribution 4.0 International License, which permits use, sharing, adaptation, distribution and reproduction in any medium or format, as long as you give appropriate credit to the original author(s) and the source, provide a link to the Creative Commons licence, and indicate if changes were made. The images or other third party material in this article are included in the article's Creative Commons licence, unless indicated otherwise in a credit line to the material. If material is not included in the article's Creative Commons licence and your intended use is not permitted by statutory regulation or exceeds the permitted use, you will need to obtain permission directly from the copyright holder. To view a copy of this licence, visit http://creativecommons.org/licenses/by/4.0/.

\section{References}

1. Acerbi, E., Buttazzo, G.: Reinforcement problems in the calculus of variations. Ann. Inst. H. Poincaré Anal. Non Linéaire 3, 273-284 (1986)

2. Ambati, V.R., Asheim, A., van den Berg, J.B., van Gennip, Y., Gerasimov, T., Hlod, A., Planqué, B., van der Schans, M., van der Stelt, S., Vargas Rivera, M., Vondenhoff, E.: Some studies on the deformation of the membrane in an RF MEMS switch. In: Proceedings of the 63rd European Study Group Mathematics with Industry, O. Bokhove, J. Hurink, G. Meinsma, C. Stolk, and M. Vellekoop, eds., CWI Syllabus, Netherlands, 1 2008, Centrum voor Wiskunde en Informatica, pp. 65-84. http:// eprints.ewi.utwente.nl/14950 (2008)

3. Banasiak, J., Roach, G.F.: On corner singularities of solutions to mixed boundary-value problems for second-order elliptic and parabolic problems. Proc. R. Soc. Lond. A 433, 209-217 (1991)

4. Bernstein, D.H., Guidotti, P.: Modeling and analysis of hysteresis phenomena in electrostatic zipper actuators. In: Proceedings of Modeling and Simulation of Microsystems 2001, Hilton Head Island, SC, 2001, pp. 306-309 (2001)

5. Dal Maso, G.: An Introduction to $\Gamma$-Convergence. Progress in Nonlinear Differential Equations and Their Applications, vol. 8. Birkhäuser Boston Inc., Boston (1993)

6. Gilbarg, D., Trudinger, N.S.: Elliptic Partial Differential Equations of Second Order. Classics in Mathematics, Springer-Verlag, Berlin, 2001. Reprint of the 1998 edition (1998)

7. König, H.: Ein einfacher Beweis des Integralsatzes von Gauß. Jber. Deutsch. Math.-Verein. 66, 119-138 $(1963 / 1964)$ 
8. Laurençot, Ph., Nik, K., Walker, Ch.: Energy minimizers for an asymptotic MEMS model with heterogeneous dielectric properties. arXiv:2003.14000 (2020)

9. Laurençot, Ph., Walker, Ch.: Some singular equations modeling MEMS. Bull. Am. Math. Soc. (N.S.) 54, 437-479 (2017)

10. Laurençot, Ph., Walker, Ch.: Shape derivative of the Dirichlet energy for a transmission problem. Arch. Rational Mech. Anal. 237, 447-496 (2020)

11. Leoni, G.: A First Course in Sobolev Spaces. Graduate Studies in Mathematics, vol. 181, 2nd edn. American Mathematical Society, Providence, RI (2017)

12. Lindsay, A.E., Lega, J., Glasner, K.G.: Regularized model of post-touchdown configurations in electrostatic MEMS: Equilibrium analysis. Physica D 280, 95-108 (2014)

13. Lindsay, A.E., Lega, J., Glasner, K.G.: Regularized model of post-touchdown configurations in electrostatic MEMS: Interface dynamics. IMA J. Appl. Math., 80, 1635-1663 (2015)

14. Maz'ya, V.G., Netrusov, Y.V., Poborchiı̌, S.V.: Boundary values of functions from Sobolev spaces in some non-Lipschitzian domains. Algebra Anal. 11, 141-170 (1999)

15. Nečas, J.: Les Méthodes Directes En Théorie Des Equations Elliptiques. Masson et Cie, Éditeurs, Paris; Academia, Éditeurs, Prague (1967)

16. Pelesko, J.A., Bernstein, D.H.: Modeling MEMS and NEMS. Chapman \& Hall/CRC, Boca Raton, FL (2003)

17. Yang, Y., Zhang, R., Zhao, L.: Dynamics of electrostatic microelectromechanical systems actuators. J. Math. Phys. 53, 022703 (2012)

Publisher's Note Springer Nature remains neutral with regard to jurisdictional claims in published maps and institutional affiliations. 